Para enlazar con este artículo / To link to this article:

http://dx.doi.org/10.14198/fem.2019.34.07

Para citar este artículo / To cite this article:

Arráez Llobregat, José Luis. «Los relatos testimoniales de mujeres francesas judías durante la Shoá. Estado de la cuestión entre el final de la guerra y finales de siglo». En Feminismo/s, 34 (diciembre 2019): 143-173. Dosier monográfico: Estado actual de la investigación en Literatura francesa y Género: balance y nuevas perspectivas / État présent de la recherche en Littérature française et Genre: bilan et nouvelles perspectives, coord. Ángeles Sirvent Ramos, DOI: 10.14198/fem.2019.34.07

\title{
LOS RELATOS TESTIMONIALES DE MUJERES FRANCESAS JUDÍAS DURANTE LA SHOÁ. ESTADO DE LA CUESTIÓN ENTRE EL FINAL DE LA GUERRA Y FINALES DE SIGLO
}

\author{
THE TESTIMONIAL ACCOUNTS OF FRENCH JEWISH \\ WOMEN DURING THE HOLOCAUST. STATE OF \\ THE QUESTION BETWEEN THE END OF THE WAR AND \\ THE END OF THE CENTURY
}

\author{
José Luis ARrÁEZ LlOBREGAT \\ Universidad de Alicante, Alicante \\ jl.arraez@ua.es \\ https://orcid.org/0000-0001-8155-1450
}

\section{Resumen}

Nuestro objetivo es mostrar y explicar la evolución de la participación de la mujer francesa judía en la memoria de la Shoá a través de la literatura testimonial en el periodo comprendido entre el final de la guerra y el fin del milenio. A tal fin, y siguiendo un criterio secuencial basado en las diferentes etapas que enmarcan el reconocimiento de la memoria de la Shoá en Francia, hemos detallado el volumen de publicaciones y reediciones. Hemos justificado su mayor o menor presencia en función de unos criterios de orden histórico, ideológico, social y académico. Paralelamente, nos hemos adentrado en la literatura testimonial de autoría masculina y en los testimonios de la Resistencia con el fin de obtener más información.

Palabras clave: Shoá; Francia; mujer; testimonial; estudios de género. (CC BY 4.0)

Feminismo/s 34, diciembre 2019, pp. 143-173 


\begin{abstract}
Our goal is to show and explain the evolution of the participation of French Jewish women in the memory of the Holocaust through testimonial literature in the period between the end of the war and the end of the millennium. To this end, and following a sequential criterion based on the different stages that frame the recognition of the memory of the Holocaust in France, we have detailed the volume of publications and reissues. We have justified its greater or lesser presence according to a criteria based on historical, ideological, social and academic terms. In parallel, we have entered the testimonial literature of male authorship and the testimonies of the Resistance in order to obtain more information.
\end{abstract}

Keywords: Holocaust; France; woman; testimonial stories; gender studies.

Las mujeres judías, independientemente de su nacionalidad, edad y condición social, padecieron indiscriminadamente las consecuencias extremas de la política antisemita nacionalsocialista, tanto en Francia, país que nos ocupa, como en la totalidad de los países invadidos. La cuestión del sexo no fue un factor beneficioso y de exclusión susceptible de tener en cuenta, cuando de lo que se trataba era, tal y como proclamó H. Himmler en Poznań, de «faire disparaître ce peuple de la Terre» (167). No obstante, el progresivo acercamiento de los estudios de género a los relatos testimoniales de las víctimas ha revelado un conjunto de especificidades propias de cada sexo. Si bien el proceso de deshumanización elaborado carecía de orientación sexual, este generaba una gran diversidad de situaciones que afectaban directamente a la naturaleza y a la cultura de las mujeres judías -religiosas o laicas- de forma distinta a las de los hombres. Pese a ello, el camino hacia el reconocimiento de una especificidad en la deshumanización de la mujer judía durante la Shoá ha sido largo en Europa, América e Israel, y no exento de un acalorado debate donde participan diferentes áreas de conocimiento y facciones del movimiento feminista.

Efectivamente, la realidad histórica fue idéntica para hombres y mujeres; dicho de otro modo, y siguiendo a M. Bártolo, D. Feierstein, G. Levy y D. Montero (25-41), en tiempos del genocidio, la «construcción de la otredad negativa», el «hostigamiento social y estatal», la «segregación social», el «debilitamiento sistemático»y el «exterminio» fueron los mismos para 
un sexo y otro; no obstante, si la realidad biológica, cultural y social de las mujeres judías es diferente a la de los hombres, es lógico considerar que las consecuencias fueran distintas, que su deshumanización fuera otra. Siguiendo la reivindicación de una revisión de la Historia desde una perspectiva de género, Dalia Ofer y L. Weitzaman afirman:

Bien qu'ils aient planifié de tuer tous les Juifs, les nazis, notamment au cours des premières années de la guerre, imposèrent des règlements et exigences de travail différents pour les hommes et pour les femmes, les deux sexes subissant des contraintes différentes. Alors qu'ils tentaient de gérer le cataclysme auquel ils étaient confrontés, les hommes et les femmes réagirent à la persécution nazie en faisant appel à des compétences et à des ressources propres à chacun des sexes (5).

Las intervenciones de las diferentes oleadas de los estudios de género han sido necesarias para identificar y mostrar la situación específica de la mujer judía durante la Shoá.

El objetivo de la investigación llevada a cabo, y cuyos resultados se exponen en este artículo de carácter descriptivo-analítico, es mostrar la evolución de la participación de las mujeres francesas judías represaliadas en la literatura de la memoria de la Shoá a través de la publicación de sus vivencias en forma de relato testimonial ${ }^{1}$. A tal fin, y con el objeto de reflejar secuencialmente los resultados, estructuraremos el artículo en tres apartados que remiten internamente a la política de reconocimiento institucional de la memoria de la Shoá en Francia concebida por Philippe Mesnard (11-19). No abordaremos el último periodo (2000-2019) denominado «la globalización» de la Shoá. Un primer acercamiento al conjunto de relatos testimoniales publicados en estas dos últimas décadas eleva la cifra por encima de las 700 publicaciones. Tal volumen precisaría un estudio particularizado que reflejara el conjunto de factores que explican semejante desarrollo en torno a la memoria de la

1. Con el fin de ofrecer una relación lo más exhaustiva posible de relatos testimoniales publicados por mujeres, pero igualmente de autoría masculina ya sean judíos o resistentes de ambos sexos, hemos consultado múltiples bases de datos de bibliotecas, centros de documentación e instituciones oficiales. No obstante, somos conscientes de la posibilidad de que haya determinadas omisiones, pues hemos constatado que no siempre se indica la fecha de la edición princeps de un determinado relato en un catálogo. Este es pues un trabajo abierto que quizás pueda enriquecerse con nuevos hallazgos.

Feminismo/s 34, diciembre 2019, pp. 143-173 
Shoá, un periodo donde participan los relatos testimoniales de la primera, segunda y tercera generación ${ }^{2}$.

En esta línea citaremos a Liliane Kandel, haciendo nuestro su proyecto: «Il s'agit [...] de rassembler enfin des souvenirs, des témoignages, des réflexions sur ce que fut le sort des femmes durant la longue nuit nazie, textes souvent inédits ou tombés dans l'oubli» (223).

Previamente utilizábamos la expresión «hacer comprensible lo incomprensible» con el fin de señalar el interés mostrado desde diferentes disciplinas que integran las humanidades por analizar, entender y difundir lo ocurrido. Al respecto destaca la actividad desarrollada por un gran número de historiadoras, quienes, frente a la primera generación integrada por historiadores y proyectada hacia relatos de autoría masculina, optan por los relatos de autoría femenina. Acerca de ello, Goldschläger et Lemaire refieren: «les historiens de la shoah appartenant à la première génération ont en partie privilégié les récits d'homme mûr, alors que les études plus récentes, souvent réalisées par la nouvelle génération d'historiennes, valorisent les témoignages féminins, qui rappellent davantage l'expérience individuelle des victimes» (78).

\section{EL RELATO DE LA MUJER FRANCESA JUDÍA EN EL SILENCIO Y LA INVISIBILIDAD DE LAS VÍCTIMAS (1945-FINALES DE LOS 60)}

Más allá de las estadísticas, de sus cifras y porcentajes elaborados fundamentalmente por sociólogos, la literatura testimonial pone rostro y da voz a las mujeres judías que en aquel entonces vivieron tanto en la Francia ocupada como en la de Vichy. No obstante, muchos de estos relatos han quedado ensombrecidos durante décadas por aquellos de autoría masculina, así como por aquellos que fueron redactados por los integrantes de la Resistencia, ya fueran hombres o mujeres. En relación con ello, Joan Ringelheim sugería en 1992 que la historia del genocidio judío se estaba realizando, concediendo un espacio exclusivo a los relatos testimoniales de hombres como Primo Levi, Elie Wiesel, Aron Appelfeld o Imre Kerstez, entre otros muchos (19-23). Indudablemente, durante décadas, la voz de la mujer de la Shoá se limitó casi exclusivamente a la de una adolescente alemana llamada Ana Frank.

2. Referente a una periodización desde una perspectiva psicoanalítica, remitimos al trabajo de la profesora Amelia Peral publicado en el presente volumen.

Feminismo/s 34, diciembre 2019, pp. 143-173 
Hoy sabemos que Rutka Laskier, Mary Berg, Éva Heyman, Helga Deen, Eva Mándlová Roubíčková, Macha Rolnikaite, Lena Jedwab Rozenberg o Rywka Lipszyc, por citar solo algunos nombres, también confiaron sus reflexiones en un diario.

La voz testimonial de la mujer judía ha estado presente durante y después de la Shoá. Limitándonos a la lengua francesa, citaremos los relatos publicados inmediatamente tras la liberación referenciados infra. Todos ellos circularon prácticamente inadvertidos pese a ser los primeros textos en aproximar la persecución y deportación a la ciudadanía francesa. Similar acogida tuvieron los relatos de Louise Alcan y de Odette Elina, ambos publicados en 1947 en editoriales de pequeña extensión. En la década de los sesenta, Jacqueline Mesnil-Amar, Madeleine Sabine y Aline-Mathilde Fathaud publicarán sus testimonios con similar recepción. Ofreceremos a continuación la relación completa de relatos siguiendo el orden cronológico de publicación:

- Julie Crémieux Dunand, La Vie des internés (1945)

- Denise Aimé, Relais des errants (1945)

- Anónimo, Birkenau: bagne de femmes par le Matricule 55.310 (1945) El relato pertenece a una mujer judía de 22 años en 1945, deportada en el convoy 58 el 31 de julio de 1943.

- Suzanne Birnbaum, Une française juive est revenue (1946, 1989)

- Dounia Ourisson, Les secrets du bureau politique d'Auschwitz (1946)

- Louise Alcan, Sans armes et sans bagages (1947)

- Odette Elina, Sans fleurs ni couronnes, avec 12 dessins originaux de l'auteur (1947, 1982, 2005)

- Luce Retten, Juive ou française? (vol. 1 publicado en 1951, vol. 2 en 1952)

- Jacqueline Mesnil-Amar, Ceux qui ne dormaient pas, 1944-1946, fragments de journal $(1957,2008,2009,2010)$

- Madeleine Sabine, Changer le sable en or (1961)

- Aline-Mathilde Fathaud, J'avais vingt ans (1968)

Incluiremos en este primer listado la recopilación de testimonios de Claudette Bloch, de Eugène Aronéanu y uno colectivo prologado por Prosper Alfaric donde participan varias mujeres supervivientes: 
Los relatos testimoniales de mujeres francesas judías durante la Shoá.

Estado de la cuestión entre el final de la guerra y finales de siglo

- Claudette Bloch (comp.), Témoignages sur Auschwitz (1946)

- Eugène Aronéanu (comp), Camps de concentration, Témoignages recueillis par Eugène Aronéanu (1946)

- Collectif, De l'Université aux camps de concentration, Témoignages strasbourgeois (1947)

Como particularidad pondremos de relieve la pertenencia a la Resistencia de Julie Crémieux Dunand, Louise Alcan, Jacqueline Mesnil-Amar y Madeleine Sabine. No podremos concluir que el hecho de ser resistentes haya podido favorecer la posible edición o reedición de sus respectivos relatos.

En esta primera aproximación al relato testimonial de la Shoá de autoría femenina se evidencia una brecha de género prácticamente desde sus inicios, si confrontamos el número de publicaciones con las de autoría masculina ${ }^{3}$. No obstante, toda explicación es insuficiente si no se indicara la gran dificultad que supuso la publicación de sus relatos para unas y otros en un país

3. Con el fin de visualizar la diferencia, procedemos a señalar los relatos testimoniales publicados de autoría masculina publicados entre 1945 y 1946. Debido al gran número de publicaciones, y con el fin de no introducir un listado excesivamente amplio, nos hemos limitado a estos dos años pues consideramos que de por sí la diferencia es evidente:

- David Benbassat (Benby D.), Je reviens du camp de Bergen-Belsen (1945)

- Marcel Conversy, Quinze mois à Buchenwald, Genève (1945)

- Jacques Darville et Simon Wichene, Drancy la Juive ou la deuxième Inquisition (1945-1946)

- Gilbert Debrise (Gilbert-Dreyfus), Cimetières sans tombeaux (1945)

- Paul Giniewski, Les Seigneurs sous la pierre (1945)

- Guy Kohen, Retour d'Auschwitz, Souvenirs du déporté 174949 (1945, 2006)

- François Kozlik, Le Mont de l'épouvante: horreurs vécues au camp du Struthof (1945)

- Eugène Minkowski, Du temps de l'étoile jaune (1945)

- José Papo, En attendant l'aurore, Activité de la Communauté Séphardite de Paris pendant l'occupation 1940-1945 (1945)

- Georges Roos, Buchenwald, Paris, Editions Médicis (1945)

- André Lettich, Trente-quatre mois dans les camps de concentration, Témoignage sur les crimes «scientifiques» commis par les médecins allemands (1946)

- Charles Papiernik, «Le 43322 raconte», Le Réveil des Jeunes, 15 juin 1945-1er juin 1946

- Boris Taslitzky, Cent onze dessins faits à Buchenwald, 1944-1945 (1946)

- Julien Unger, Le Sang et l'Or. Souvenirs de camps allemands (1946, 2007)

- Georges Wellers, De Drancy à Auschwitz (1946)

- Léon Werth, Déposition, Journal 1940-1944 (1946, 1992, 2007)

Feminismo/s 34, diciembre 2019, pp. 143-173 
cuyos intereses políticos, sociales, ideológicos y culturales estaban centrados fundamentalmente en la «reconstrucción» moral e identitaria de la nación mediante el glorioso ensalzamiento de la Resistencia. Tomaremos como punto de referencia el significativo número de relatos testimoniales publicados por resistentes francesas únicamente entre 1945 y 1946 con el fin de no ofrecer un listado extenso que nos alejaría de nuestros intereses:

- Leila de Dampierre, De l'ambassade au bagne (1945)

- Sabine Hoisne, Chambre 535, Ou mes cinq prisons pendant l'occupation (1945)

- Suzanne Normand (éd.), LibertéShip. Avec Suzanne, Alain, AnneMarie, Claude, Dormeur, François, Jacques, Pierre et Raymond ont écrit ce livre après l'avoir vécu (1945)

- Camille Folliet et Jean Folliet, Croquis de prison et Chemin de Croix inachevé (1945, 1985)

- Albertine David, Mémoires d’une internée politique (1946)

- Janine Bouissounouse, Maison occupée (1946, Gallimard reeditará hasta siete veces el relato)

- Claudette Bloch (comp.), Témoignages sur Auschwitz (1946)

- Agnès Humbert, Notre guerre, (1946, reeditado en 2004 con el título: Notre guerre: souvenirs de Résistance: Paris 1940-41, le bagne, occupation en Allemagne)

- Thérèse Mitrani, Service d'évasion (1946)

- Violette Maurice, N.N. (Nacht und Nebel) (1946, 2001, 2009).

- Suzanne Wilborts, Pour La France, Angers, La Santé, Fresnes, Ravensbrück, Mauthausen (1946.

- Suzanne Busson, Dans les griffes nazies, Angers, Fresnes, Ravensbrück, Mauthausen (1946, 1952, 2014)

En estos dos años hemos contabilizado un total de 17 publicaciones no colectivas, entre las que distinguimos 16 primeras ediciones y 2 reediciones de este mismo periodo. Por otro lado, 3 coediciones donde figuran testimonios de autoría masculina:

- Claudette Bloch (comp.), Témoignages sur Auschwitz (1946)

- Eugène Aronéanu (comp), Camps de concentration, Témoignages recueillis par Eugène Aronéanu (1946)

- Collectif, De l'Université aux camps de concentration, Témoignages strasbourgeois (1947)

Feminismo/s 34, diciembre 2019, pp. 143-173 
- Germaine Tillion, Ravensbrück, Cahiers du Rhône (1946, 1973, 1988, 1997)

- Renée Mirande-Thomas, De Ravensbrück à Mauthausen (1946)

- Albertine David, Mémoires d'une internée politique (1946)

- Noémi Fany-Lefèbvre, Six mois à Fresnes (1946)

- Alice Courouble, Amie des Juifs (1946)

- Yvonne Pagniez, Scènes de la vie du bagne (1946, 1947)

- Hélène Acard, Ma captivité en 1944, sous l'occupation allemande (1946)

- Eugène Aronéanu (comp.), Camps de concentration. Témoignages (1946)

- Anonyme, De l'Université aux camps de concentration. Témoignages strasbourgeois (1947)

- Collectif, De l'Université aux camps de concentration, Témoignages strasbourgeois (1947)

- Madeleine Fauconneau du Fresne, De l'enfer des hommes à la cité de Dieu (1947)

La diferencia es tan evidente como contundente la afirmación que efectuábamos anteriormente acerca del olvido de la Shoá a favor de la evocación de la Resistencia, puesto que el ensalzamiento de ambas realidades era inviable en plena reconstrucción del país. Del mismo modo, las editoriales y el público lector se decantaban preferentemente por los relatos de quienes habían estado en el frente, cuyas historias y adversidades resultaban mucho más sugerentes. A juicio de Goldschläger y de Lemaire:

S'il est une tradition d'écriture féminine bien ancrée depuis des siècles, c'est celle de la rédaction de journaux intimes ou de livres de confidences. Nombre de femmes vont consigner leurs expériences et leurs émotions sur le papier, mais peu d'entre elles auront le courage de les présenter à un éditeur, qui ne les accueillera d'ailleurs pas volontiers. [...] De tels récits personnels rédigés pendant la guerre verront le jour beaucoup plus tard (118).

A propósito del motivo del silencio, de la invisibilidad y de la indiferencia de la primera generación -sin distinción de género- en los años inmediatos a la liberación, Simone Veil argumenta lo siguiente:

Si nous n'avons pas parlé c'est parce que l'on n'a pas voulu nous entendre, pas voulu nous écouter. Parce que ce qui est insupportable, c'est de parler et 
de ne pas être entendu. C'est insupportable. Et c'est arrivé tellement souvent, à nous tous. Que, quand nous commençons à évoquer, que nous disons quelque chose, il y a immédiatement l'interruption. La phrase qui vient couper, qui vient parler d'autre chose. Parce que nous gênons. Profondément, nous gênons (3).

Citaremos igualmente a Andrée Majerowicz: «Les uns, au bout d'un moment, me regardaient horrifiés et comme incrédules. [...] Tout me paraissait si décalé que je croyais être devenue cinglée. Pourtant, je savais bien que non. [...] Alors j'ai pris l'habitude de me taire» (Kahn 95). Las puertas de las editoriales estaban cerradas a la par que la mirada y la escucha de sus compatriotas se dirigían hacia otros intereses. Esta actitud social no podía quizás ser otra cuando los mandos del régimen nacionalsocialista estaban siendo juzgados por las instituciones judiciales en Europa por conjura o conspiración, por crímenes contra la paz y la humanidad, así como por crímenes de guerra. En ningún momento se enjuició el genocidio judío, siendo este relegado a un segundo puesto. En este clima es lógico pensar que se obviase a la comunidad judía deportada por persecución. Por el contrario, el proceso a A. Eichmann en Israel (1961), cuyo auto acusatorio incluía quince cargos relativos a crímenes contra el pueblo judío y la humanidad, crímenes de guerra y de asociación a una organización hostil, alentó a los y las supervivientes para que compartieran sus testimonios. A ello sumaremos el impacto del juicio sobre determinadas intelectuales, véase por ejemplo Hannah Arendt (Eichmann en Jerusalén: Un estudio sobre la banalidad del mal (1963, 1. a edición francesa, 1966). Pese a ello, la relativa influencia sobre la conciencia pública de quienes sobrevivieron se refleja en el número de publicaciones localizadas entre 1960 y 1970. En el caso concreto de los relatos de autoría femenina en Francia, la práctica nula repercusión en la conciencia de las mujeres de estos juicios se constataría en el ínfimo volumen de relatos publicados entre los 60 y los 70 .

Al margen de este contexto sociopolítico con posibles efectos adversos, hay un segundo factor importante susceptible de tener en cuenta a nivel emocional y psicológico. La «parálisis de la memoria», el trauma provocado por las perniciosas experiencias vividas bloquearía el paso de la memoria individual a la memoria cultural, impidiéndose de esta forma que se efectúe el proceso resiliente en forma de testimonio postraumático. Acerca de ello y desde un punto de vista clínico, Nathalie Zajde considera que «la pathologie

Feminismo/s 34, diciembre 2019, pp. 143-173 
des survivants était une réponse congruente à ce qu'avaient cherché les bourreaux: la déflagration psychique et physique de la victime» (24).

Retomando los listados anteriores, que permiten confrontar el número de publicaciones de autoras judías frente al de autoras resistentes, la diferencia es tan elocuente como influyente es el argumento, que justificaría en parte el reducido número de autorías femeninas en el dato estadístico. Este cifra en solo un 3\% el porcentaje de deportados y deportadas francesas judías que sobrevivieron a los campos de concentración y de exterminio, siendo un 0,5\% mujeres y un 9,5\% hombres (Klarsfeld 1122-1124). Mencionaremos asimismo el argumento que relaciona el bajo número de publicaciones con la formación cultural de unas frente a otras ${ }^{4}$. Si un número destacado de supervivientes, aunque de adopción francesa, proceden de Europa del Este, es conveniente especificar que el nivel cultural de las mujeres judías depende de cada país, así pues, la realidad de los shtetls en los países del este difiere mucho de otros lugares geográficos donde las mujeres reciben la misma formación que los varones de la familia. Uno y otro influyeron ciertamente, así como en determinados casos la educación religiosa tradicional, que aumentaría su introversión, discreción y pudor referente a lo vivido durante la Shoá, frenando de esta manera su posible actividad testimonial. Por otra parte, es muy significativo exponer un criterio de tipo personal y genérico, pues tras el regreso de los campos, uno de los principales deseos, una de las prioridades de las mujeres era saberse fértiles para refundar un hogar, lo que explicaría el incremento de la natalidad desde 1947. Ello era incompatible con una escritura y unos recuerdos mórbidos que impedían ir hacia un futuro esperanzador.

El factor político e institucional expuesto anteriormente permitiría explicar el exiguo volumen de publicaciones sin establecer ninguna distinción de género, así como las copiosas publicaciones de mujeres resistentes. Por otro lado, acerca del papel que pudiera haber jugado el feminismo francés coetáneo, y que hubiera facilitado el discurso de las mujeres judías, estableceremos que sus intereses eran otros, pese a que su lucha se centraba en

4. Al respecto, citaremos a Celia S. Heller a través de D. Ofer y de L. J. Weitzman:

En Europe orientale, les femmes juives étaient aussi plus familiarisées avec le monde de la culture. Comme l'a montré Celia Heller, dans nombre de familles, en particulier de la classe moyenne, ce sont les femmes qui furent les «agents de l'acculturation», introduisant la culture polonaise à la maison et la transmettant à leurs enfants (482).

Feminismo/s 34, diciembre 2019, pp. 143-173 
el reconocimiento de los derechos civiles y políticos de las mujeres, derechos que especialmente habían sido ninguneados y violados en el caso de las mujeres judías en fechas muy recientes. Recordaremos que el movimiento feminista francés se hallaba en pleno proceso de redefinición tras los recientes cambios sociales que siguieron a la guerra; tal y como S. Van de Casteele Schweitzer sostiene, «une expression individuelle, littéraire et philosophique, [...] prend parti sans détour contre la situation des femmes telle qu'elle existe dans l'époque, essayant d'en comprendre les raisons, les conséquences et les moyens d'y remédier» (17). Sus reivindicaciones se proyectarán fundamentalmente hacia cuestiones relacionadas con el cuerpo de la mujer (sexualidad, embarazo, aborto), el trabajo y la violencia de género, que cristalizarán en la década de los 70. Al respecto, no hubo ninguna mirada retrospectiva al pasado más cercano de las mujeres que permitiera poner de manifiesto o condenar lo ocurrido durante el judeocidio.

Si hacer visible lo ocurrido resultó tan complicado que al final todo interés prácticamente se desvaneció, hacer comprensible lo incomprensible para los investigadores e investigadoras no fue mucho más sencillo, de ahí la carencia de estudios específicos centrados en la mujer judía publicados en este periodo en Francia ${ }^{5}$. En este sentido convendría señalar la ausencia de filósofas francesas judías contemporáneas a los acontecimientos que pudieran haberse interesado por el tema; citaremos, por ejemplo, a Éliane Amado Levy-Valensi, cuya madre fue deportada, a Francine Bloch o a Jeanne Hersch, suiza de expresión francesa. Esta situación cambiará, como veremos, a partir de la década de los 90.

Desde el punto de vista histórico quisiéramos resaltar el valor documental de estos primeros relatos cuyas autoras narran sus vivencias en unas condiciones exclusivas que no se reproducirán en adelante. A medida que aumenta el flujo de testimonios orales y escritos sobre la Shoá, a medida que las y los testigos se alejan de los hechos históricos, aumenta el riesgo de contaminación por otros relatos en circulación, así como por el peligro de alteración de la realidad vivida. Efectivamente, la inmediatez y la espontaneidad confieren un valor extraordinario a estas primeras publicaciones.

5. Tal y como mostraremos más adelante, en EE. UU. y en Gran Bretaña verán la luz los primeros estudios específicos. 
Si estos relatos contienen una serie de datos exclusivos que no hallamos en los ensayos históricos, en los textos firmados por mujeres encontramos en concreto una valiosa información sobre las diferentes manifestaciones de solidaridad que fortalecieron el espíritu de grupo en los guetos y en los campos, sobre la separación entre madres e hijos, sobre las reacciones biológicas ante la deshumanización física, sobre las mujeres embarazadas o sobre los abortos naturales y provocados. Tal y como Roland S. Süssmann sostiene:

Dans cet univers d'une autre planète, géré par des lois que l'esprit humain se refuse à comprendre, les femmes essayèrent de survivre en établissant un contact humain avec d'autres femmes, en formant ce qui a été appelé des «familles de substitution». Le lien qui les unissait était une soif de vie envers et contre tout (6).

En este sentido, la valía e interés de estos relatos reside en la información facilitada sobre un determinado aspecto desconocido o poco conocido, además, evidentemente, de constituir una prueba documental. Pongamos, como ejemplo, todo aquello que se refiere al «atentado» padecido contra la feminidad en los campos debido a la violación de los sentimientos de pudor y de decencia: la desnudez colectiva ante la mirada de hombres, el rasurado público del todo el pelo del cuerpo, la suciedad física -especialmente la íntima-, la desaparición de la menstruación y el temor a la esterilidad. Todo lo ocurrido en este escenario constituye una brusca ruptura relacionada con la formación familiar, religiosa -en determinados casos- y social inculcadas.

De todo ello fueron exclusivamente testigos las mujeres, al compartir un mismo espacio físico prohibido a la población masculina. Frente a aquellas y aquellos historiadores que censuran la incorporación de los relatos testimoniales en el proceso de reconstrucción de la realidad histórica (Poliakov, Hilberg), historiadores como Saul Friedländer (L'Allemagne nazie, vols. 1 y 2) estiman que estos completarían la información extraída y analizada de la documentación oficial procedente fundamentalmente de los archivos nazis. $\mathrm{Al}$ respecto, Steven T. Katz sostiene que

face à la documentation allemande et officielle en tout genre, les chercheurs commencent à saisir que, pour appréhender la nature véritable de la destruction des Juifs d'Europe, il s'avère nécessaire d'aller plus loin que la simple déconstruction sémantique des archives nazies [...] tous ensemble, [...] parviennent à composer une mosaïque qui offre une peinture distincte de la 
réalité de la Shoah, laquelle ne peut se découvrir ou trouver son équivalent dans toute autre source (7).

Haremos extensibles estas consideraciones a los relatos publicados en los sucesivos periodos en los que se desarrolla la memoria de la Shoá; evidentemente, una vez comprobada la autenticidad de este y el respeto a las coordenadas históricas. Por otro lado, es fundamental ser consciente de que se trata de un documento personal, sujeto a una posible fragmentariedad y subjetividad frente a la realidad histórica.

Más allá del valor documental de estos primeros relatos, quisiéramos resaltar el gran valor personal de estas primeras relatoras para quienes encerrarse en el silencio hubiera supuesto un encarcelamiento postconcentracionario. La palabra escrita se convirtió para todas ellas en un instrumento de salvación mediante el cual efectuaron su proceso catártico.

\section{LA CONTRIBUCIÓN DE LOS RELATOS DE LA MUJER JUDÍA A LA «MÉMORIALISATION» DE LA SHOÁ (DÉCADA 70-80)}

El «silencio» y la «invisibilidad» de la Shoá se prolongarán hasta finales de la década de los 60. Nuevamente, será el contexto sociopolítico el que determine la evolución de la memoria de la Shoá en estas dos décadas. Se produce un silencio absoluto hasta la década de los 70 , periodo en el que se reanuda la publicación de relatos.

Hemos localizado 3 publicaciones colectivas dirigidas por hombres y en las que participan mujeres:

- Henri Moraud (éd.), Jawischowitz, une annexe d'Auschwitz, 45 déportés, 8 mineurs polonais témoignent (1985)

- David Diamant (éd), Par-delà les barbelés, Lettres jetées des trains de déportation, écrits d'Auschwitz, créations journalistiques, littéraires et artistiques (1986)

- Collectif, Séance solennelle de témoignages, 1942, Paris, Palais du Luxembourg, 8 novembre 1987 (1989)

Sobre un total de 29 obras no colectivas publicadas y localizadas en este periodo, distinguimos: un relato mixto (autor y autora), 24 primeras ediciones 
de autoría femenina, 3 reediciones de este mismo periodo, 2 reediciones del periodo anterior 6 :

- Marcelle Christophe et Christophe, Robert, Le Miracle de nos prisons, 1940-1945 (1974)

- Marcelle Christophe, Le miracle de nos prisons: 1940-1945 (1974)

- Denise Baumann, Une famille comme les autres, Lettres réunies et présentées par Denise Baumann... (1973, 1985)

- Estrea Zaharia Asseo, Les Souvenirs d'une rescapée (1974)

- Anne Hélène Marie Katz-Codaccioni, Les Cahiers de Marie-Hélène (1975)

- Fania Fénelon, Sursis pour l'orchestre. Témoignage recueilli par Marcelle Routier (1976, reeditado dos veces en 1982 en France loisirs y en Le Grand livre du mois)

- Renée Louria, Les Russes sont à Lemberg (1979)

- Clara Malraux,... Et pourtant j'étais libre (1979, 2006)

- Louise Alcan, Le temps écartelé. Récit (1980)

- Hélène Stark, Mémoires d’une juive hongroise: 1940-1945 (1981)

- Éva Baer, Le Serre kiki (1981)

- Ginette Hirtz, Les Hortillonnages sous la grêle, Histoire d'une famille juive en France sous l'Occupation (1982)

- Camille Touboul, Un Relais d'étoiles: Souvenirs d'Auschwitz (1982)

- Liliane Babitcheff, Les Aventures d'une Autrichienne pendant l'Occupation (1982)

- Frida Weinstein, J'habitais rue des Jardins Saint-Paul (1983)

- Roma Elster, 28, rue Nowolipki: Varsovie 1939-1945 (1983)

- Irène Durst, La Famille Moszkowicz (1984)

- Doudou Gentille Cohen, J'ai vécu Auschwitz (1985)

- Odette Fabius, Un lever de soleil sur le Mecklembourg: mémoires (1986)

- Monique Lévi, Mon étoile (1986)

- Yvonne Wurzel-Bauh, Lombre des camps (1987)

- Eva Tichauer, J'étais le numéro 20832 à Auschwitz (1988)

- Rosine Mayan, Pour que jamais tu n'oublies (1989)

6. Suzanne Birnbaum, Une française juive est revenue (1989) y Odette Elina, Sans fleurs ni couronnes, avec 12 dessins originaux de l'auteur (1982). 
- Louise Jacobson, Lettres de Louise Jacobson, 1er septembre 1942-13 février 1943, Jeune lycéenne... emprisonnée à Fresnes, internée à Drancy, déportée à Auschwitz parce qu'elle était juive... (1989, 1997)

El volumen de publicaciones de mujeres es superior -casi se duplica- frente al periodo anterior, igualándose al número de relatos de autoría masculina, como podrá comprobarse a continuación. Sin embargo, hemos constatado que el volumen de ediciones de autoría masculina se mantiene, no habiendo una diferencia visible respecto del periodo anterior como en el caso de las autorías femeninas.

Señalaremos nuevamente las 3 obras colectivas:

- Henri Moraud (éd.), Jawischowitz, une annexe d'Auschwitz, 45 déportés, 8 mineurs polonais témoignent (1985)

- David Diamant (éd), Par-delà les barbelés, Lettres jetées des trains de déportation, écrits d'Auschwitz, créations journalistiques, littéraires et artistiques (1986)

- Collectif, Séance solennelle de témoignages, 1942, Paris, Palais du Luxembourg, 8 novembre 1987 (1989)

Hay un total de 29 publicaciones, entre las que distinguimos: una obra mixta, 23 primeras ediciones, cuatro reediciones de este mismo periodo, una reedición del periodo anterior ${ }^{7}$ :

- Claude Vigée, La Lune d'hiver. Récit - journal - essai (1970, 2002)

- Sim Kessel, Pendu à Auschwitz (1970, 1974)

- Maurice Bakcha, Les Grooms de Lucifer (1972)

- Georges Wellers, L'Étoile jaune à l'heure de Vichy: de Drancy à Auschwitz (1973)

- Pierre Cahen, Le Médecin malgré eux (1972)

- Joseph Joffo, Un Sac de billes (1973, 1974, 1978, 1975, 1973, 1974, 1974, 1976)

- Philippe Erlanger, La France sans étoile, Souvenirs de l'avant-guerre et du temps de l'occupation (1974)

7. Henry Bulawko, Les Jeux de la mort et de l'espoir, Auschwitz-Jaworzno (1980).

Feminismo/s 34, diciembre 2019, pp. 143-173 
- Marcelle Christophe et Robert Christophe, Le Miracle de nos prisons, 1940-1945 (1974)

- Max Wolfshaut-Dinkes, Échec et mat: récit d'un survivant de Pchemychl en Galicie (1975, 1983 y 1985)

- Roger Gompel, Pour que tu n'oublies pas (1975)

- Charles Lopata, J'ai survécu (1977)

- Paul Chitelman, Ceux et celles qui m'ont fait homme, Chronique de mes rencontres (1977)

- René Brivet, Carnets de guerre, 1940-1945 (1978)

- Saul Friedländer, Quand vient le souvenir (1978, 1981, 1998)

- Georges Wellers, La Solution finale et la mythomanie néo-nazie (1979)

- Georges Wellers, Les Chambres à gaz ont existé: des documents, des témoignages, des chiffres (1981)

- Maurice Rajsfus, Quand j'étais juif (1982)

- Chimon Hammel, Frédéric, «ouviens-toi d'Amalek», Témoignage sur la lutte des Juifs en France, 1938-1944 (1982)

- Raymond Raoul Lambert, Carnet d’un témoin: 1940-1943 (1984)

- Lionel Rocheman, La Belle âge ou les Douceurs de la guerre (1984)

- Raymond Wachtenheim, La vie à tout prix (1984)

- Adam Rayski, Nos illusions perdues (1985)

- Jack Kuper, Jack Kuper (1985)

- René Samuel Kapel, Un rabbin dans la tourmente, Dans les camps d'internement et au sein de l'Organisation juive de Combat, 1940-1944 (1986)

- Sylvain Kaufmann, Au-delà de l'enfer (1987)

- Sam Hoffenberg, Le camp de Poniatowa: la liquidation des derniers Juifs de Varsovie (1988)

- André Balbin, De Lodzà Auschwitz, En passant par la Lorraine (1989)

- Max Heilbronn, Galeries Lafayette, Buchenwald, Galeries Lafayette (1989)

Aunque no expongamos los resultados detenidamente, resulta interesante destacar la brecha de género existente entre los relatos testimoniales de varones represaliados y detenidos por su militancia en la Resistencia o por su 
actividad política y los de mujeres represaliadas y detenidas por idénticos motivos $^{8}$.

El reconocimiento de la década de los 70 y de los 80 como las de la «mémorialisation» (Peschanski 7) de la Shoá es el resultado de la conjunción de una serie de acciones cuyo resultado será la singularización del acontecimiento histórico, la configuración de la memoria del judeocidio como «asunto» público de interés nacional, así como el reconocimiento del estatus social de la víctima y de su trauma. Determinados acontecimientos históricos y sociales, así como su importante mediatización, ayudan a comprender el paso del silencio a la palabra escrita y oral en Francia. Del mismo modo, el trabajo en común de historiadores e historiadoras, testigos y asociaciones sobre la memoria de la Shoá será decisivo para una primera ruptura con el aislamiento social de las víctimas. Mencionaremos especialmente la gran actividad social realizada por Beate y Serge Klarsfeld desde finales de los 60: publicación de Mémorial de la déportation des Juifs de France (1978) y creación en 1979 de la fundación Fils et filles de déportés juifs de France.

Destacaremos, por encima de otros, la persecución, detención y procesamiento de Paul Touvier (1989-1992), de Maurice Papon (1981-1998) y de Klaus Barbie (1983-1987), retransmitidos por televisión. A ello se añade la emisión de la miniserie americana Holocausto (1979) y del documental Shoah de Cl. Lanzmann (1985) por televisión. Gracias a la mediatización de la Shoá, muchas de las víctimas, hasta el momento «aisladas», empatizarán con las y los testigos de las respectivas acusaciones, así como con las víctimas entrevistadas por Lanzmann para su documental. Nos referiremos, por último, al impacto en las y los supervivientes de las publicaciones negacionistas de Robert Faurisson desde finales de los 70. Todo ello favorecerá considerablemente la toma de la palabra oral y escrita para narrar sus vivencias. Del mismo modo que Simone Lagrange y Charlotte Wardi habían sido capaces de testificar, ellas podrían igualmente testificar en forma de relato testimonial escrito lo vivido. En este clima cómplice, afable y protector, las víctimas se transformaron en portadoras de una nueva ética democrática basada en los derechos humanos y en la convivencia (Wieviorka 41-42).

8. Sobre un total de 216 publicaciones localizadas: 20 pertenecen a mujeres, hay 3 obras mixtas, 18 son colectivas y el resto son de autoría masculina.

Feminismo/s 34, diciembre 2019, pp. 143-173 
Este contexto revela cómo progresivamente el tema se abre paso en la conciencia de las diferentes autoridades y de un potencial público lector. Todo ello derivará en la evolución de la conciencia de los y las supervivientes acerca del rol que su testimonio como víctimas del genocidio ocupa en la sociedad. Ciñéndonos a la autoría femenina, las primeras publicaciones ejercieron, sin lugar a duda, un efecto de llamada sobre determinadas supervivientes, impulsando el número de publicaciones. Adicionalmente, el todavía modesto, pero significativo volumen de relatos testimoniales de mujeres publicados en este segundo periodo podría hallar su explicación en la influencia del movimiento feminista francés, especialmente activo desde mayo del 68. Tras la consecución de los derechos civiles y políticos, el feminismo de «segunda generación» orientó su lucha hacia la liberalización del cuerpo de la mujer. Destacaremos una de las acciones sociales más mediáticas y de mayor repercusión social realizada a pie de calle. El 26 de agosto de 1970 un grupo de mujeres, acompañadas de pancartas con el lema «Hay alguien más desconocido que el soldado desconocido, su esposa», depositaron bajo el Arco de Triunfo en París, una corona con la dedicatoria «a la esposa del soldado desconocido». Acciones como esta pudieron influir en el despertar de la conciencia de las mujeres y en la necesidad de narrar sus experiencias, fundamentalmente las concentracionarias, pues hemos observado que los relatos se centran especialmente en el proceso de deshumanización padecido en los campos.

Decididamente, el contexto político e ideológico animó a traspasar -aun así, insuficientemente- la barrera entre lo privado y lo público a las supervivientes, especialmente a aquellas con una destacada militancia política y social como Fania Fénelon, exintegrante de la Resistencia, o como Renée Louria y Eva Tichauer, ambas afiliadas al partido comunista. Influidas por el dinamismo y el entusiasmo de su activismo, estas se involucraron en la construcción de la memoria histórica de la Shoá en Francia a través de la publicación de sus relatos.

La difusión de sus vivencias se halla igualmente impulsada por el deseo de difundir la historia del genocidio judío acallada hasta el momento. Acerca de la importancia de los escritos íntimos, mencionaremos a Georges Gusdorf: «le témoin s'efface dans son témoignage; il ne prétend être qu'un médiateur, un révélateur de cette vérité en gestation, de cette gestation de vérité à laquelle il lui est donné d'assister» (263). Estos relatos ofrecen datos esenciales

Feminismo/s 34, diciembre 2019, pp. 143-173 
sobre la persecución y deportación de las mujeres judías; por otro lado, sus descripciones sobre la cotidianeidad y sus reflexiones sobre la guerra nos acercan a una realidad en una sociedad tradicional patriarcal donde prevalecen la distribución de tareas por sexo. Referente a ello, Roland S. Süssmann argumenta que:

Les femmes juives vivaient dans une société essentiellement conservatrice et patriarcale; les hommes étaient les chefs de famille tandis que les femmes remplissaient leur fonction traditionnelle au foyer ou aidaient leurs pères/ maris à gagner leur vie. Par conséquent, elles n'avaient aucun rôle dans la direction ou la guidance de la communauté. Pendant la Shoah toutefois, elles ont assumé un rôle familial capital qui peut être défini comme étant «l'affirmation de la vie»: la tentative de survivre dans n'importe quelle situation (2).

Depositarios de una exquisita valía documental, a través de los relatos es posible reconstruir la historia de las mujeres durante la Shoá.

No insistiremos en este segundo apartado en el valor documental de estos relatos donde narran desde el punto de vista de una mujer judía-laica o religiosa- el ritual de deshumanización que les arrebató su identidad humana y sexual. Asimismo, incidiremos en que hemos partido del conocimiento de que el funcionamiento del sistema concentracionario es el mismo para hombres y mujeres. Como lo hace notar Anne Postel-Vinay, «tous les détenus subissaient en principe le même régime d'humiliation, de cruauté et d'acheminement vers la mort» (4); sin embargo, puntualizará:

Le système concentrationnaire n'était donc pas sexuellement orienté, du moins pas plus que ne l'étaient les conceptions générales de l'époque, mais à l'intérieur du cadre général commun, le monde qu'il produisait présentait une grande diversité de situations (5).

Creemos conveniente mencionar el nacimiento y desarrollo de los estudios de género aplicados a la Shoá, un hecho científico en el que participan diferentes áreas de conocimiento, y que alejará Francia de los EE. UU., de Alemania, de Gran Bretaña y de Israel, países donde los Gender Studies of the Holocaust surgen en los años 70-80. El interés de detenernos en estos, exponiendo brevemente sus principales líneas de pensamiento, reside en las críticas que surgirán en Francia en la década de los 90 proponiendo nuevos planteamientos.

Durante las décadas de los 70 y de los 80 , el deseo de «hacer comprensible lo incomprensible» da un giro al penetrar los Gender Studies od the

Feminismo/s 34, diciembre 2019, pp. 143-173 
Holocaust en el Holocausto en EE. UU. y en Alemania. En 1983, Joan M. Ringelheim (United States Holocaust Memorial Museum) organiza el primer encuentro científico de esta nueva realidad científica ( Women surviving the holocaust») que dará lugar en 1985 al ensayo editado conjuntamente con Esther Katz: Proceedings of the Conference, Women Surviving the Holocaust (1983). Desde una perspectiva humanística y social, el objetivo prioritario consistía en mostrar la especificidad de la represión y de la deportación de las mujeres judías durante la Shoá. En 1984 se publica el ensayo dirigido por las historiadoras germanistas Renate Bridenthal, Atina Grossman y Marion Kaplan, When Biology became Destiny. Women in Weimar and Nazi Germany. El conjunto de las investigaciones, centradas especialmente en el estudio de las mujeres «arias» bajo el nacionalsocialismo, específicamente en la familia y en la reproducción, conceden sin embargo muy poco espacio a las mujeres alemanas judías.

Nos detendremos brevemente en la enunciación de los principales postulados de este periodo, pues serán utilizados a posteriori en Francia desde la institución universitaria por determinadas investigadoras como Liliane Kandel. Partiendo del principio de «la diferencia», de que las mujeres judías fueron objeto de una doble persecución, como mujeres y como judías, J.M. Ringelheim considera que los estudios de género deben resaltar la especificidad de los mecanismos de persecución padecidos por las mujeres judías y poner de manifiesto la particularidad de las diferentes modalidades de supervivencia y de resistencia desarrolladas en los guetos. Los primeros trabajos de J. M. Ringelheim, alentados por el «feminismo cultural», se asientan sobre el principio de la «invisibilidad» testimonial de la mujer judía perseguida y concentrada, debido a la sobrevaloración de los relatos firmados por hombres. Atribuye un papel importante a la recusación de la tesis de la «unicidad» que defiende que todos los grupos humanos fueron por igual víctimas del nazismo, y a la defensa de la tesis de «diferencia», según la cual las mujeres poseen unas vivencias específicas derivadas de su cuerpo y de su sexo. En un segundo periodo, e influenciada por el «feminismo radical» $\mathrm{y}$ «el nacionalismo femenino», Ringelheim proyecta un estudio de la mujer judía durante la Shoá bajo la óptica de la opresión y de la dominación por el hombre, sin que se matice el término «nazi». Previamente a su distanciamiento de la esfera académica, Ringelheim desarrolla, en un tercer periodo, una serie de

Feminismo/s 34, diciembre 2019, pp. 143-173 
conclusiones a partir del uso de estadísticas referidas a las mujeres en los guetos, en los convoyes de deportación y en los campos.

En cambio, Francia, país cuyo colaboracionismo con el régimen nazi comenzó tras la reunión entre el Mariscal Pétain y Adolph Hitler el 24 de octubre de 1940, dando así comienzo a la Francia de Vichy, no se aproximará a esta realidad científica desde una perspectiva de género hasta la década de los 90 .

Por último, y formando parte de este deseo de «hacer comprensible lo incomprensible», pero no desde una perspectiva de género, mencionaremos la obra de las historiadoras Nadine Fresco, Renée Poznanski y de Annette Wieviorka. La germanista Rita Thalmann constituye una excepción con su ensayo Etre femme sous le III ${ }^{e}$ Reich (1982).

\section{LA ACTIVIDAD TESTIMONIAL DE LA MUJER FRANCESA JUDÍA EN LA «CONMEMORACIÓN» DE LA SHOÁ (DÉCADA DE LOS 90)}

En la década de los 90 se produce un importante vuelco político y judicial que insuflará una energía decisiva, especialmente a los supervivientes, para contribuir al denominado «travail de mémoire» ${ }^{9}$ de la Shoá. El 28 de julio es declarado por François Mitterrand en 1993 JOURNÉE NATIONALE À LA MÉMOIRE DES VICTIMES DES CRIMES RACISTES ET ANTISÉMITES DE L'ÉTAT FRANÇAIS ET D'HOMMAGE AUX JUSTES DE FRANCE. No obstante, el reconocimiento y el autoinculpamiento de Francia y de su administración en la persecución de la comunidad francesa judía se verbaliza el 16 de julio de 1995, en el discurso de Jacques Chirac ante el monumento conmemorativo del 53. ${ }^{\circ}$ aniversario de «la rafle du Vélodrome d'Hiver». Mencionaremos el inicio en 1997 del proceso a Maurice Papon, tras 17 años de procedimiento judicial, en el que testimonian un gran número de víctimas. Como ocurriera con el proceso Klaus Barbie, la proyección mediática del juicio, así como de los acontecimientos anteriores señalados, serán decisivos para la toma de la palabra oral y escrita de quienes fueron víctimas del estado de Vichy, para transformar la imposición del «devoir de l'oubli» en «devoir de mémoire».

9. Si bien inicialmente se utilizará la noción de «devoir de mémoire», posteriormente (año 2015 aproximadamente) se prefiere «travail de mémoire».

Feminismo/s 34, diciembre 2019, pp. 143-173 
Durante esta década, en general, el volumen de publicaciones es tímidamente superior respecto al periodo anterior, abriéndose una importante brecha entre las publicaciones de un sexo y otro.

Hemos localizado 6 publicaciones colectivas, entre las que predominan las ediciones dirigidas por mujeres:

- Annette Kahn (éd), Personne ne voudra nous croire (1991)

- Elisabeth Marum-Lunau (éd), «Boches ici, Juifs là-bas». Correspondance d'exilés du IIIe Reich, 1939-1942 (1997)

- Karine Habif (éd), Le jour d'après. Douze témoins de la libération des camps (1995)

- Myriam Foss et Lucien Steinberg (éds), Vie et mort des Juifs sous l'Occupation. Récits et témoignages (1996)

- Lucien Steinberg et Myriam Foss (éds), Opération «Vent printanier». 16-17 juillet 1942 la rafle du Vél'd'Hiv (1992)

- Serge Ejnès (éd), Histoire des Juifs de Reims pendant la Seconde guerre mondiale. Témoignages, documents, déportés (1995)

Sobre un total de 49 publicaciones no colectivas localizadas en este periodo, distinguimos: 33 primeras ediciones de autoría femenina, 15 reediciones de este mismo periodo y una reedición del periodo anterior ${ }^{10}$ :

- Renée Roth-Hano, Touchons du bois! (dos reediciones en 1990)

- Annette Muller, La Petite fille du Vel'd'Hiv'. Récit (1991, 1992, 1999, 2008, 2009, y dos veces en 2012)

- Fela Brajtberg-Fajnzylber, Le témoignage ordinaire d'une juive polonaise (1991)

- Forrester Viviane, Ce soir, après la guerre (1992, dos veces 1993, 1997)

- Nadine Heftler, Si tu t'en sors... Auschwitz, 1944-1945 (1992)

- Liliane Lévy-Osbert, Jeunesse vers l'abîme (1992)

- Sabine Zlatin, Mémoires de la «dame d'Izieu» (1992)

- Annette Zaidman, Le train de la mémoire: Drancy-Auschwitz, 19421992 (1992)

- Alice Steinitz, Quitte ton pays (1993)

10. Marcelle Christophe et Robert Christophe, Une famille dans la guerre. 1940-1945 (1994). 
- Nadine Bitner, Papa va revenir (1993, reeditado una segunda vez en 1993 y en 1995)

- Franka Oppenheim, Adieu Alma-Ata (1993, 1996, 1997)

- Sarah Kofman, Rue Ordener, Rue Labat (1994)

- Charlotte Schapira, Il faudra que je me souvienne. La déportation des enfants de l'Union générale des Israélites de France (1994)

- Sabine Zlatin, Les enfants d'Izieu. Suivi du témoignage de Sabine Zlatin (1994)

- Odette Abadi, Terre de détresse. Birkenau, Bergen-Belsen, Médecin, résistante juive et survivante d'Auschwitz et de Bergen-Belsen (1995, 2012)

- Ida Akerman-Tiéder, Et tu raconteras à tes enfants... (1995)

- Vivette Samuel, Sauver les enfants (1995)

- Juliane Picard, Des ténèbres à la lumière. D'Auschwitz-Birkenau au lac Majeur (1995, 1999, 2007, 2008)

- Denise Holstein, Je ne vous oublierai jamais, mes enfants d'Auschwitz. Témoignage (1995)

- Francine Christophe, Une petite fille privilégiée (1996, 2001, 2014)

- Micheline Larès-Yoël, France 40-44. Expérience d'une persécution (1996)

- Françoise Maous, Coma Auschwitz, n. ${ }^{\circ}$ A.5553. Récit (1996)

- LisaDrach, Les fantômes de Lisa (1996)

- Odile Grand, Couleur citron, côté cœur (1996, 1997, 1998)

- Juliette Bénichou, Comme la paille dans le vent. Journal d'une jeune fille juive $(1997,1998)$

- Danièle Gervais-Marx, La Ligne de démarcation (1997, 2004)

- Simone Lagrange, Coupable d'être née. Adolescente à Auschwitz (1997)

- Évelyne Krief, Une enfance interdite ou la petite marrane (1997)

- Liliane Lelaidier-Márton, À l'ombre de l'étoile. Réminiscences d'une enfant cachée (1997, 2006)

- Éliane Le Fournis, Le présage de la rose (1998)

- Denise Geskis, Pluie et vents sur une étoile (1999)

- Rachel Perlstein, Exode et résistance en Savoie (1999)

- Myriam Ben, Quand les cartes sont truquées. Mémoires (1999) 
Por lo que respecta a las publicaciones de autor, hemos localizado 6 publicaciones colectivas (mencionadas también anteriormente):

- Annette Kahn (éd), Personne ne voudra nous croire (1991)

- Elisabeth Marum-Lunau (éd), «Boches ici, Juifs là-bas». Correspondance d'exilés du IIIe Reich, 1939-1942 (1997)

- Karine Habif (éd), Le jour d'après. Douze témoins de la libération des camps (1995)

- Myriam Foss et Lucien Steinberg (éds), Vie et mort des Juifs sous l'Occupation. Récits et témoignages (1996)

- Lucien Steinberg et Myriam Foss (éds), Opération «Vent printanier». 16-17 juillet 1942 la rafle du Vél'd'Hiv (1992)

- Serge Ejnès (éd), Histoire des Juifs de Reims pendant la Seconde guerre mondiale. Témoignages, documents, déportés (1995)

Hemos localizado igualmente 2 publicaciones en la que colaboran dos autores:

- Jacques Eidiliman et Albert Eidiliman, Deux rescapés du génocide racontent (1991)

- Georges Kohn et François Montel, Journal de Compiègne et de Drancy (1999)

El total de primeras publicaciones es de 70, entre las que distinguimos: 55 primeras ediciones, 12 reediciones de este mismo periodo y 3 reediciones del periodo anterior ${ }^{11}$ :

- Robert Weil, Témoignage (1990)

- Bernard Fride, Une mauvaise histoire juive (1991)

- Etienne Rosenfeld, De Drancy à ces camps dont on ne parle pas (1991)

- Georges Wellers, Un Juif sous Vichy (1991)

- Jacques Biélinky, Un journaliste juif à Paris sous l'Occupation. Journal: 1940-1942 (1992)

- Sylvain Kaufmann, Le livre de la mémoire. Au-dela de l'enfer (dos ediciones en 1992)

11. Léon Werth, Déposition. Journal 1940-1944 (1992); Fred Sedel, Habiter les ténèbres, Auschwitz, Jawozno, Birkenau, Oranienburg, Sachsenhausen, Landsberg, Kaufering (1990); Saul Friedländer, Quand vient le souvenir (1998).

Feminismo/s 34, diciembre 2019, pp. 143-173 
- Maurice Rajsfus, Jeudi noir. La rafle du 16 juillet 1942 (1992)

- Jo Amiel, La Rafle. Un sana très ordinaire, 1942-1944 (1993)

- René Brivet, J'ai échappé aux nazis. Journal d'un jeune Alsacien aux trois quarts juif, 20 juin 1940-10 septembre 1944 (1993)

- Lucien Duckstein, Les Guerres sont loin (1993)

- Joseph Farnel, La Ballade du petit Joseph (1993)

- Robert Ichah, Le judéo-maso. L'itinéraire d'un enfant né sous une étoile jaune (1993)

- Maurice Schiff, Histoire d'un bambin juif sous l'Occupation nazie. 1942, 3 ans miraculé! (1993, 2005)

- Maurice Obréjan, L'étrange destinée d’un homme trois fois français (1994)

- Jérôme Scorin, Litinéraire d'un adolescent juif. De 1939 à 1945 (1994)

- Léon Arditti, Vouloir vivre. Deux frères à Auschwitz... (1995)

- Herman Berger, Carnets d'un médecin de montagne. Les mémoires du docteur Berger $(1995,2011)$

- Henry Bily, Destin à part. Seul rescapé de la rafle de Clans du 25 octobre 1943 (1995)

- Paul Cerf, Dégagez-moi cette racaille (1995)

- Armand Guilbert, Kaddish pour les miens. Chronique d'un demi-siècle d'antisémitisme (1995, 1998)

- Willy Holt, Femmes en deuil sur un camion (1995)

- Jean-Claude Moscovici, Voyage à Pitchipoï (1995, 1996, 1997, 2009, 2016)

- Claude-Henri Mouchnino, Survivant par hasard (1995)

- Lucien Nach, Un couple, une famille sous l'Occupation (1995)

- Nathan Prochownik, Mémoires barbelées. Et après... (1995)

- Isaac Schoenberg, Lettres à Chana. Camp de Pithiviers: Mai 1941-juin 1942 (1995)

- Henri Tajchner, Trois ans dans l'enfer d'Auschwitz. Parole de survivant (1995)

- Pierre Vidal-Naquet, Mémoires. 1. La brisure et l'attente, 1930-1955 (1995)

- Armand Guilberg, Kaddish pour les miens. Chronique d'un demi-siècle (1995, 1998) 
- Lazare Domniez, Voyage à travers la mémoire d'un enfant. 1942-1945. Récit (1996)

- Yves Lejoyeux, La vie à contresens. 1938-1944 (1996)

- Joseph Gourand, Les cendres mêlées. Récit (1996, 1997)

- Albert Wilkowsky, De l'étoile jaune au drapeau rouge (1996, 1998, 2007).

- Maurice Ajzen, Il n'y a pas de saison pour la mort. Maurice Ajzen raconte Auschwitz-Birkenau, Varsovie, Dachau... (1997)

- Emile Copfermann, Dès les premiers jours de l'automne (1997)

- Robert Francès, Un déporté brise son silence (1997)

- François Stupp, Réfugié au pays des Justes. Araules, 1942-1944 (1997)

- David Szlamowicz, Un enfant dans la tourmente. La dangereuse aventure d'un adolescent sous l'Occupation (1997)

- Bernard Teperman, Passé décomposé ou La spirale d’un passé (1997)

- Camille Touboul, Le Plus long des chemins de Marseille à Auschwitz. De Marseille à Auschwitz (1997)

- Max Weinstein, «Souvenirs, souvenirs» (1997)

- Charles Fichter, Entre parenthèses. Souvenirs (1998)

- Roger François, Ses portes resteront fermées la nuit car il n'y aura pas de jours. Dachau, 21 octobre 1944-21 avril 1945 (1998)

- Léon Grynberg, Mémoires de Léon Grynberg, rescapé d'Auschwitz. 19031979 (1998)

- Louis Aron, Journal de Louis Aron, directeur de la Maison israélite de refuge pour l'enfance. Neuilly-sur-Seine, 1939, Crocq (Creuse), 19391942, Chaumont (Creuse), 1942-1944 (1998)

- Léon Lerher, 172749. Un Poulbot à Pitchipoï (1998)

- Maurice Rajsfus, 10 ans en 1938. Souvenirs d'enfance à l'ombre du donjon de Vincennes (1998)

- Alain Sené, Des millions de Riskine: extraits du journal, 1939-1945 (1998)

- Jacques Stroumsa, Tu choisiras la vie. Violoniste à Auschwitz (dos ediciones en 1998)

- Dominique Laury, Un hiver à voix basse. Récit (dos ediciones en 1998, 1999)

- Léon Poliakov, L’auberge des musiciens. Mémoires (1999) 
- Maurice Cling, Vous qui entrez ici. Un enfant à Auschwitz (1999)

- Joseph Gourand, Renaître à la vie (1999, 2000)

- Victor Sullaper, Vigtor le rebelle. La résistance d'un Juif en France: récit biographique (1999)

- StanislasTomkiewicz, L'adolescence volée (1999, 2001)

Tal y como puede constatarse, el número de publicaciones de mujeres es relativamente superior respecto a la década anterior; no obstante, el número de relatos de autores casi se quintuplica. Ni el contexto político favorable ni el avance de los estudios de género parecen haber favorecido el aumento de testimonios de autoría femenina. Recordaremos que, en Francia, durante la década de los 90, «la troisième vague du féminisme» avanza entre disensiones, centrando su lucha a nivel institucional en cuestiones como la paridad, la erradicación de las desigualdades o el desarrollo de los estudios de género.

En la década de los 90 las investigaciones prosiguen en EE. UU. entre debates y polémicas ${ }^{12}$. Israel, en colaboración con EE. UU., se inicia en estos estudios entre 1994-1995. Destacaremos especialmente la colaboración entre Dalia Ofer y L. Weitzman cuyo resultado es Women in the Holocaust (1998). Tal y como hemos indicado en nota a pie de página, no desarrollaremos este punto pues nos alejaríamos del tema que nos ocupa. Apuntaremos nuevamente el recurso a este material en las investigaciones desarrolladas en Francia a partir del 2000.

En Francia, y con carácter tardío, estos estudios se inician en el ámbito universitario en la década de los 90 desde diferentes áreas de conocimiento. Especialmente interesantes son los trabajos de la socióloga Liliane Kandel en los que efectúa una severa crítica al posicionamiento teórico de Ringelheim. Considera que la filósofa norteamericana se ha servido de la Shoá para censurar el patriarcado:

12. Abordar el conjunto de los estudios realizados desde EE. UU., Alemania, Gran Bretaña, Austria o Israel nos alejaría del tema que nos ocupa. Al respecto remitimos al estudio realizado por Isabelle Ernot.

Ernot sintetiza y analiza contrastivamente cuáles han sido los principales ejes sobre los que se ha vertebrado la construcción de unos estudios de género específicos aplicados a la investigación de la mujer judía durante la Shoá. Lo importante es resaltar que todos ellos influirán en el desarrollo de las investigaciones desarrolladas en Francia.

Feminismo/s 34, diciembre 2019, pp. 143-173 
Mais, précisément pour cette raison, il n'est pas acceptable de la voir instrumentalisée, manipulée, mise au service d'une démonstration qui lui est totalement étrangère. Car contrairement aux dénégations de principe de l'auteure, il ne s'agit pas de décrire, aussi fidèlement que possible, la vie, l'expérience, la mort enfin des femmes durant les temps de catastrophe; ni même d'examiner l'impact complexe de genre, les modulations diverses, multiples, des rapports entre hommes et femmes dans les situations de privation, d'humiliation ou de danger extrême où ils se trouvent pris. Non, ce n'est malheureusement pas de cela qu'il s'agit, mais au contraire de mettre avant -voire de construireles seuls éléments susceptibles d'argumenter et d'étayer une thèse: celle de l'universalité de l'oppression patriarcale, de la domination des femmes par les hommes, des bénéfices constamment accumulés par ceux-ci y compris au prix du malheur et de la souffrance de celles-là (230).

Por otro lado, desde la Historia citaremos el ensayo de Francine Muel-Dreyfus, Vichy et l'éternel féminin (1996).

No obstante, ello no parece haber infundido el suficiente coraje a las supervivientes para hacer uso de la palabra escrita u oral con el fin de transmitir sus vivencias. El optimismo de los años anteriores se desvanece. Cierto es que aumenta el número de publicaciones, no así proporcionalmente frente a las de autoría femenina.

Esta tendencia cambiará bruscamente con la llegada del nuevo milenio y la denominada «globalización» de la Shoá pues alcanza el plano institucional a nivel mundial: la conferencia internacional sobre la Shoá celebrada en Estocolmo el 28 de enero de 2000 adoptará una declaración solicitando la apertura de todos los archivos e insistiendo en la necesidad de educar a las futuras generaciones. Por otro lado, el 1 de noviembre de 2005 la Asamblea General de la ONU decidió por consenso designar el 27 de enero DÍA INTERNACIONAL DE CONMEMORACIÓN ANUAL DE LAS VÍCTIMAS DEL HOLOCAUSTO, para recordar la liberación de los campos de concentración nazis en Europa, en 1945. La resolución insta a los Estados miembros a elaborar programas educativos que inculquen a las generaciones futuras las enseñanzas del Holocausto para prevenir actos de genocidio. A nivel interno, tras el discurso de Chirac, diferentes homólogos reconocen la implicación del estado en la deportación de miembros de la comunidad judía francesa. Destaca especialmente la creación de LA FONDATION POUR LA MÉMOIRE DE LA SHOAH en 2000 bajo la iniciativa de Alain Juppé.

Feminismo/s 34, diciembre 2019, pp. 143-173 
Bajo el nuevo contexto político y social, cientos de supervivientes francesas toman la iniciativa de narrar la Shoá.

\section{CONCLUSIONES}

Con el objetivo de analizar la evolución de la toma de la palabra escrita de la mujer francesa judía durante la Shoá y así poder visibilizarla, nos hemos adentrado igualmente en los relatos de autoría masculina, incluso en los relatos de los y las integrantes de la Resistencia. Esto nos ha permitido obtener una mejor comprensión del lugar que ocupa el relato testimonial de la mujer francesa judía como parte de periodo histórico y literario concreto, y no como un fragmento aislado. Al poner de relieve las tres realidades literarias señaladas, situadas en un mismo espacio y en un mismo tiempo, simultáneamente hemos podido extraer una serie de conclusiones tras acudir a un conjunto de explicaciones de orden histórico, cultural, ideológico y académico. La presentación de estos relatos nos ha permitido, por un lado, mostrar una realidad editorial y literaria, reflejo del acontecer de la Historia, de quienes la «construyen» y «circulan» por ella; por otro lado, y quizás sea lo más importante, esta búsqueda en bases de datos y catálogos nos ha permitido contribuir a la difusión de una realidad histórica insuficientemente conocida hasta hace relativamente poco tiempo. Acercarse a la persecución y a la deportación a través de este valioso material desde una perspectiva de género, no significa defender que la persecución y deportación de las mujeres fuera mayor y en peores condiciones frente a la de los hombres. Defendemos que, si bien el objetivo último para unas y otros fue la deshumanización, esta se experimentó de forma distinta en función del género. La defensa de esta realidad sería inviable si no tuviéramos a nuestra disposición estos relatos en cuyo contenido hallamos los rastros sobre una deshumanización que en ciertos aspectos diferenció a mujeres y hombres, pero que indudablemente, y por encima de todo, unió a ambos. 


\section{REFERENCIAS BIBLIOGRÁFICAS}

Bard, Christine. «L'histoire des femmes au défi de la déportation». Histoire@ Politique 2.5 (2008): 1-20.

Bártolo, Marcela, Daniel Feierstein, Guillermo Levy, y Damián Montero. Seis estudios sobre genocidio. Análisis de relaciones sociales: otredad, exclusión, exterminio. Daniel Feierstein. Buenos Aires: Editores del Puerto, 2000.

Dray-Bensousan, Renée. «La Shoah et l'institutionnalisation du devoir de mémoire». Controverse 2 (2006): 67-81

Ernot, Isabelle. ««Women and/in the Holocaust»»: à la croisée des Women'sGender et Holocaust Studies (Années 1980-2010)». Genre \& Histoire 15 (Automne 2014-Printemps 2015).

Friedländer, Saul. L'Allemagne nazie et les Juifs. Tome 1, Les années de persécution, 1933-1939. Paris: Seuil, 1997.

Friedländer, Saul. L'Allemagne nazie et les Juifs. Tome 2, Les années d'extermination, 1939-1945. Paris: Seuil, 2008.

Goldschläger, Alain, et Jacques Lemaire. Les témoignages écrits de la Shoah. Bruxelles: Racine, 2016.

Gusdorf, Georges. Lignes de vie. Les écritures du moi. Paris: Odile Jacob, 1991.

Hilberg, Raoul. The Destruction of the European Jews. Chicago: Quadrangle, 1961. Himmler, Heinrich. Discours secrets. Ed Bradley F. Smith y Agnès F. Peterson. Paris: Gallimard, 1978.

Kahn, Annette. Personne ne voudra nous croire. Paris: Payot, 1991.

Kandel, Liliane. «Une pensée empêchée: des usages du genre et de quelques-unes de ses limites». Les Temps Modernes 587 (1996): 220-248.

Katz, Esther, and Joan Miriam Ringelheim. Eds. Proceedings of the Conference, Women Surviving the Holocaust, New York: Institute for Research in History, 1983.

Klarsfeld, Serge. Le calendrier de la persécution des Juifs en France. Paris: FFDJF et The Beate Klarsfeld Foundation, 1993.

Mesnard, Philippe. Consciences de la Shoah. Critique des discours et des représentations, Paris: Kimé, 2000.

Ofer, Dalia, et Lenore J. Weitzman. Sur la spécificité de la place des femmes dans la Shoah. Fondements théoriques d'une analyse. Traduit de l'anglais par Claire Drevon. Revue d'Histoire de la Shoah 1.188 (2008): 479-506.

Peschanski, Denis. Dir. Mémoire et mémorialisation. V. 1: de l'absence à la représentation, Paris, Hermann, coll. «Mémoire(s)», 2013. 
Poliakov, Léon. Le Bréviaire de la haine. Le IIIe Reich et les Juifs, préface de François Mauriac. Paris: Calmann-Lévy, 1951.

Postel-Vinay, Anise. «Camps d'hommes, camps de femmes: premières approches. Étude d'une ancienne déportée de Ravensbrück». Histoire@Politique 2.5 (2008): 1-12.

Ringelheim, Joan. «The Holocaust. Taking women into account». Jewish Quaterly 39.3 (1992): 19-23.

«Simone Veil et la parole refusée des déportés», L'Histoire en direct, 02/05/1988. https://www.franceculture.fr/histoire/simone-veil-sur-la-shoah-nous-navonspas-parle-parce-quon-na-pas-voulu-nous-ecouter. 1 de junio de 2019.

Süssmann, Roland S. «Les femmes dans la Shoah». Shalom. Le magazine juif européen Automne 2007, 6. http://www.shalom-magazine.com/Article. php?id=480111. 6 de junio de 2019.

Van De Casteele Schweitzer, Sylvan. «Le mouvement féministe en France après la deuxième guerre mondiale». Matériaux pour l'histoire de notre temps 1 (1985): 15-19.

Wieviorka, Annette. L'Ère du témoin. Paris: Hachette Pluriel, 1998.

Zajde, Nathalie. Guérir de la Shoah. Paris: Odile Jacob, 2005. 